02

\title{
Исследование магнитных свойств ферроборатов неодима и самария методом спектроскопического эрбиевого зонда
}

\author{
() Е.П. Чукалина ${ }^{1}$, А. Яблуновский ${ }^{2}$, И.А. Гудим ${ }^{3}$ \\ ${ }^{1}$ Институт спектроскопии РАН, \\ 108840 Троицк, Москва, Россия \\ ${ }^{2}$ Московский фризико-технический институт (национальный исследовательский университет), \\ 141701 Долгопрудный, Россия \\ ${ }^{3}$ Институт фризики им. Л.В. Киренского Сибирского отделения РАН, \\ 660036 Красноярск, Россия \\ e-mail: echukalina@isan.troitsk.ru
}

Поступила в редакцию 22.07.2021 г.

В окончательной редакции 22.07.2021 г.

Принята к публикации 07.08.2021 г.

\begin{abstract}
Методом эрбиевого спектроскопического зонда исследованы ферробораты $\mathrm{NdFe}_{3}\left(\mathrm{BO}_{3}\right)_{4}$ и $\mathrm{SmFe}_{3}\left(\mathrm{BO}_{3}\right)_{4}$, активированные $1 \%$ эрбия, со структурой хантита (пространственная группа симметрии $R 32$ ). Из анализа температурной зависимости спектров пропускания в области перехода ${ }^{4} I_{15 / 2} \rightarrow{ }^{4} I_{13 / 2}$ в ионе $\mathrm{Er}^{3+}$ установлено, что оба исследуемых соединения антиферромагнитно упорядочиваются при $T_{\mathrm{N}} \approx 33 \mathrm{~K}$ в легкоплоскостную магнитную структуру. Не обнаружено других фазовых переходов.
\end{abstract}

Ключевые слова: мультиферроики, оптическая спектроскопия, кристаллы с редкими землями, крамерсовские ионы.

DOI: $10.21883 /$ OS.2022.01.51896.23-21

\section{Введение}

Метод эрбиевого спектроскопического зонда (см., например, $[1,2])$ был разработан в группе М.Н. Поповой и успешно применен для исследования магнитных фазовых переходов и типов магнитных структур в нескольких семействах изоструктурных оксидных соединений, а именно в „голубых“ $\left.R_{2} \mathrm{Cu}_{2} \mathrm{O}_{5}[3,4]\right)$ и „зеленых“ $R_{2} \mathrm{BaCuO}_{5}$ [5-7]) сопутствующих фазах высокотемтературных сверхпроводников типа 1-2-3, в никелатах $R_{2} \mathrm{BaNiO}_{5}$ [8-11] (здесь $R-$ редкоземельный (Р3) элемент или иттрий). В работах [12-14] этим методом были исследованы также соединения $R \mathrm{Fe}_{3}\left(\mathrm{BO}_{3}\right)_{4}(R=\mathrm{Pr}$, $\mathrm{Eu}-\mathrm{Er}, \mathrm{Y})$ из семейства P3 ферроборатов со структурой хантита и получена информация о типах реализующихся в них магнитных структур. Эта информация была позже подтверждена результатами нейтронных экспериментов. Исследования методом эрбиевого зонда для ферроборатов неодима и самария, $\mathrm{NdFe}_{3}\left(\mathrm{BO}_{3}\right)_{4}$ и $\mathrm{SmFe}_{3}\left(\mathrm{BO}_{3}\right)_{4}$, до сих пор не проводились. Они выполнены в настоящей работе.

\section{Информация о структуре и свойствах $\mathrm{NdFe}_{3}\left(\mathrm{BO}_{3}\right)_{4}$ и $\mathrm{SmFe}_{3}\left(\mathrm{BO}_{3}\right)_{4}$}

Ферробораты неодима и самария кристаллизуется в ромбоэдрической сингонии с пространственной группой симметрии R32. Кристалл $\mathrm{SmFe}_{3}\left(\mathrm{BO}_{3}\right)_{4}$ антиферромагнитно упорядочивается при температуpe $T_{\mathrm{N}}=32 \pm 1 \mathrm{~K}$ [15-17]. Комплексные исследования магнитных, магнитоупругих и магнитоэлектрических свойств $[17,18]$ и спектроскопическое исследование ориентированных монокристаллов $\mathrm{SmFe}_{3}\left(\mathrm{BO}_{3}\right)_{4}$ [15] показали, что ниже температуры Нееля $T_{\mathrm{N}}$ моменты ионов $\mathrm{Fe}^{3+}$ упорядочиваются в $a b$ плоскости, перпендикулярной оси $c$ кристалла. Легкоплоскостной характер антиферромагнитной структуры был подтвержден в экспериментах по рассеянию нейтронов [19]. Магнитные свойства ферробората самария были объяснены на основе параметров кристаллического поля и параметров обменного взаимодействия между Р3 ионами и ионами железа [20]. Параметры были получены из экспериментального и теоретического исследования штарковской структуры мультиплетов иона $\mathrm{Sm}^{3+}$ в кристаллах $\mathrm{SmFe}_{3}\left(\mathrm{BO}_{3}\right)_{4}$ в парамагнитной и магнитоупорядоченной фазах [20].

Исследование температурной зависимости магнитной восприимчивости кристалла $\mathrm{NdFe}_{3}\left(\mathrm{BO}_{3}\right)_{4}$, проведенное в работе [21], показало наличие максимума при $32 \mathrm{~K}$, связанного с антиферромагнитным упорядочением подсистемы ионов железа, и особенности при $6 \mathrm{~K}$. Авторы предположили, что низкотемпературная особенность связана с трехмерным антиферромагнитным упорядочением как в подрешетке $\mathrm{Fe}^{3+}$, так и в подрешетке $\mathrm{Nd}^{3+}$. Позже в спектроскопическом исследовании $\mathrm{NdFe}_{3}\left(\mathrm{BO}_{3}\right)_{4}$ [22] было установлено магнитное упорядочение при $33 \mathrm{~K}$, а особенность при $6 \mathrm{~K}$ связана с перераспределением населенностей расщепленных крамерсовских дублетов иона $\mathrm{Nd}^{3+}$. В работе [23] по исследованию магнитных и термодинамических свойств 
монокристалла $\mathrm{NdFe}_{3}\left(\mathrm{BO}_{3}\right)_{4}$ с учетом спектроскопических данных показано, что низкотемпературная особенность при $6 \mathrm{~K}$ на температурной зависимости магнитной восприимчивости и аномалия Шоттки при $4 \mathrm{~K}$ на температурной зависимости теплоемкости связаны не с магнитным упорядочением Р3 подсистемы, а с перераспределением населенностей компонент крамерсовских дублетов иона $\mathrm{Nd}^{3+}$, расщепленных обменным взаимодействием. На основе анализа температурного поведения широкодиапазонных спектров пропускания в линейно-поляризованном свете в области переходов в ионе $\mathrm{Nd}^{3+}$ в кристалле $\mathrm{NdFe}_{3}\left(\mathrm{BO}_{3}\right)_{4}$ был выполнен расчет по теории кристаллического поля (КП) [24]. Из расчета были получены, в частности, физически обоснованные параметры КП, а также волновые функции и $g$-факторы для штарковских уровней иона $\mathrm{Nd}^{3+}$. Результаты расчета указывают на первостепенную роль магнитной анизотропии Р3 ионов в установлении того или иного типа магнитной структуры [24].

Что касается исследований магнитной структуры $\mathrm{NdFe}_{3}\left(\mathrm{BO}_{3}\right)_{4}$, то в опубликованных статьях встречаются противоречивые данные. В работе [25] проводились измерения магнитной восприимчивости и теплоемкости, рентгеноструктурные исследования и эксперименты по дифракции неполяризованных нейтронов на порошковых и монокристаллических образцах ферробората неодима. Было определенно, что при $1.6 \mathrm{~K}$ магнитный момент подсистемы ионов $\mathrm{Fe}^{3+}$ составляет $4.9 \mu \mathrm{B}$, что близко к моменту свободных ионов железа. Магнитный момент подсистемы ионов $\mathrm{Nd}^{3+}$, по-видимому, насыщается из-за эффектов кристаллического поля, достигая $2.7 \mu \mathrm{B}$. Магнитные измерения указывают на антиферромагнитное упорядочение при температуре Нееля $30.5 \mathrm{~K}$. В работе [25] было также обнаружено резкое увеличение магнитного момента подсистемы ионов $\mathrm{Nd}^{3+}$ примерно при $15 \mathrm{~K}$, которое предположительно связали с фазовым переходом из соразмерной в несоразмерную фазу. В работе [26] было проведено исследование монокристалла $\mathrm{NdFe}_{3}\left({ }^{11} \mathrm{BO}_{3}\right)_{4}$ при помощи нейтронографии и сферической нейтронной поляриметрии. Результаты нейтронографии показывают, что ниже $T_{\mathrm{N}}=30 \mathrm{~K}$ магнитные моменты $\mathrm{Fe}^{3+}$ и $\mathrm{Nd}^{3+}$ лежат в базисной плоскости $a b$, упорядочены ферромагнитно в одной плоскости и антиферромагнитно в соседних вдоль оси $c$ плоскостях. В работе [26] уточняется, что ниже температуры $T_{\mathrm{IC}}=13.5 \mathrm{~K}$ магнитная структура переходит в несоразмерную антиферромагнитную спираль вдоль оси $c$ с периодом примерно $1140 \AA$.

\section{Эксперимент}

Исследуемые в настоящей работе монокристаллы $\mathrm{NdFe}_{3}\left(\mathrm{BO}_{3}\right)_{4}: \mathrm{Er}$ (1 at.\%) были выращены растворрасплавным методом с использованием флюса на основе $\mathrm{Bi}_{2} \mathrm{Mo}_{3} \mathrm{O}_{12}$ [27]. Выращивание велось в групповом режиме на затравках, полученных предварительно в режиме спонтанного зарождения. Кристаллодержатель с четырьмя затравками опускался в тигель с растворрасплавом и вращался с заданной частотой $30 \mathrm{rpm}$. Таким образом, процесс роста длился от 10 до 15 суток, при этом температура раствор-расплава понижалась с нарастающим темпом $0.04-0.125 \mathrm{~K} / \mathrm{h}$, так чтобы скорость роста кристаллов не превышала $1 \mathrm{~mm}$ в сутки. Аналогичным методом, но на основе флюса $\mathrm{Li}_{2} \mathrm{WO}_{4}$, были получены монокристаллы $\mathrm{SmFe}_{3}\left(\mathrm{BO}_{3}\right)_{4}: \mathrm{Er}$ (1 at.\%). Выращенные монокристаллы имели хорошее оптическое качество и зеленую окраску, характерную для ферроборатов. Из них были вырезаны и отполированы неориентированные пластинки толщиной $2.2 \mathrm{~mm}$ и $4.42 \mathrm{~mm}$ для $\mathrm{NdFe}_{3}\left(\mathrm{BO}_{3}\right)_{4}: \mathrm{Er}\left(1\right.$ at.\%) и $\mathrm{SmFe}_{3}\left(\mathrm{BO}_{3}\right)_{4}: \operatorname{Er}(1$ at.\%) соответственно.

Спектры поглощения регистрировались на фурье-спектрометре Bruker IFS 125 HR в спектральной области от 5000 до $10000 \mathrm{~cm}^{-1}$ со спектральным разрешением до $0.1 \mathrm{~cm}^{-1}$. Температурные измерения проводились как с помощью оптического заливного гелиевого криостата, так и криостата замкнутого цикла Cryomech ST 403 в диапазоне температур от 4 до $300 \mathrm{~K}$. Контроль и стабилизация температуры образца, находящегося в криостате, осуществлялись через двухканальный термоконтроллер Scientific Instruments 9700 .

\section{Результаты и обсуждение}

На рис. 1 изображены спектры пропускания кристалла $\mathrm{NdFe}_{3}\left(\mathrm{BO}_{3}\right)_{4}: \mathrm{Er}^{3+}(1 \mathrm{at} \%)$ в области переходов со штарковских уровней основного мультиплета ${ }^{4} I_{15 / 2}$ иона $\mathrm{Er}^{3+}$ на штарковские уровни возбужденного мультиплета ${ }^{4} I_{13 / 2}$ при четырех температурах $(50,31,20$ и $5 \mathrm{~K})$.

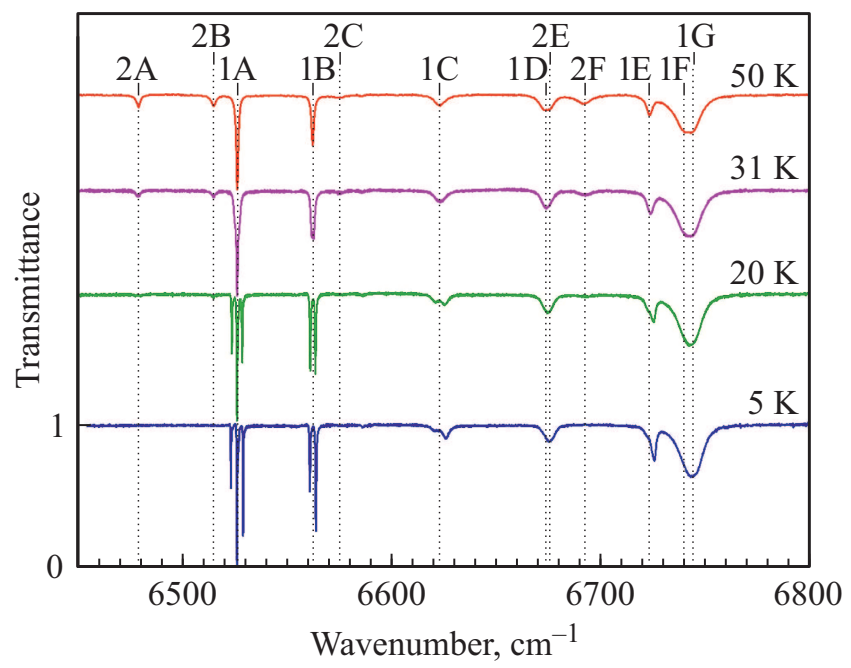

Рис. 1. Спектры пропускания кристалла $\mathrm{NdFe}_{3}\left(\mathrm{BO}_{3}\right)_{4}: \mathrm{Er}^{3+}$ $\left(1\right.$ at.\%) в области переходов ${ }^{4} I_{15 / 2} \rightarrow{ }^{4} I_{13 / 2}$ в ионе $\mathrm{Er}^{3+}$. Спектры при разных температурах смещены по вертикали друг относительно друга. Обозначения спектральных линий приведены в соответствии со схемой на рис. 2. 


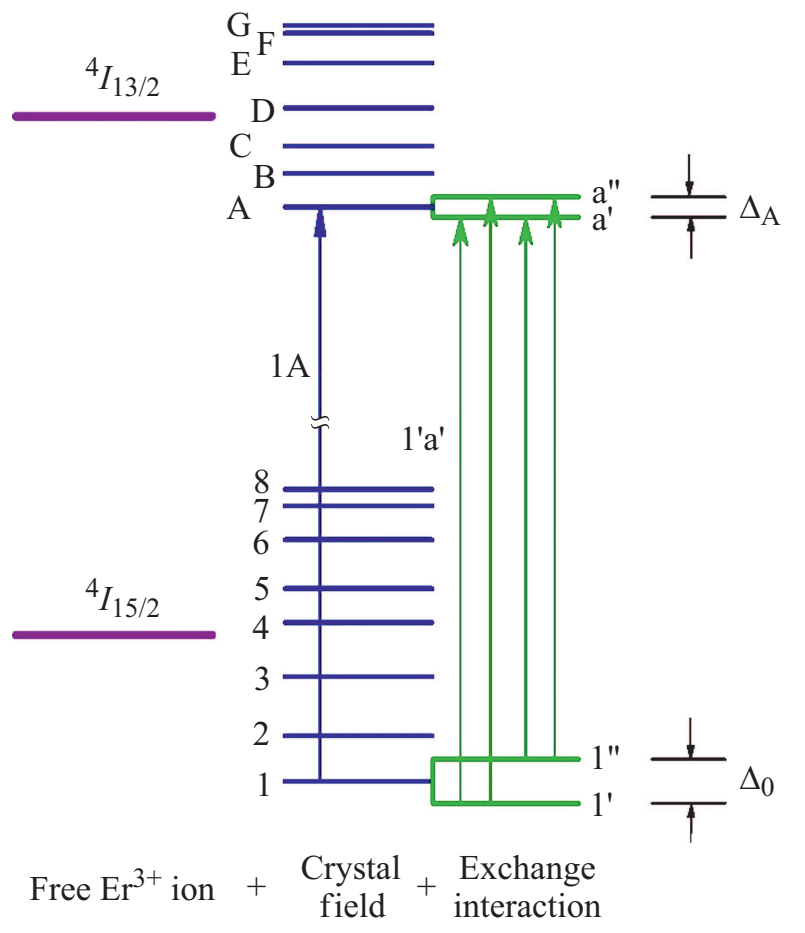

Рис. 2. Схема штарковских уровней основного ${ }^{4} I_{15 / 2}$ и возбужденного ${ }^{4} I_{13 / 2}$ мультиплетов иона $\mathrm{Er}^{3+}$ в кристаллическом поле и обменные расщепления крамерсовских дублетов в магнитоупорядоченном состоянии кристалла.

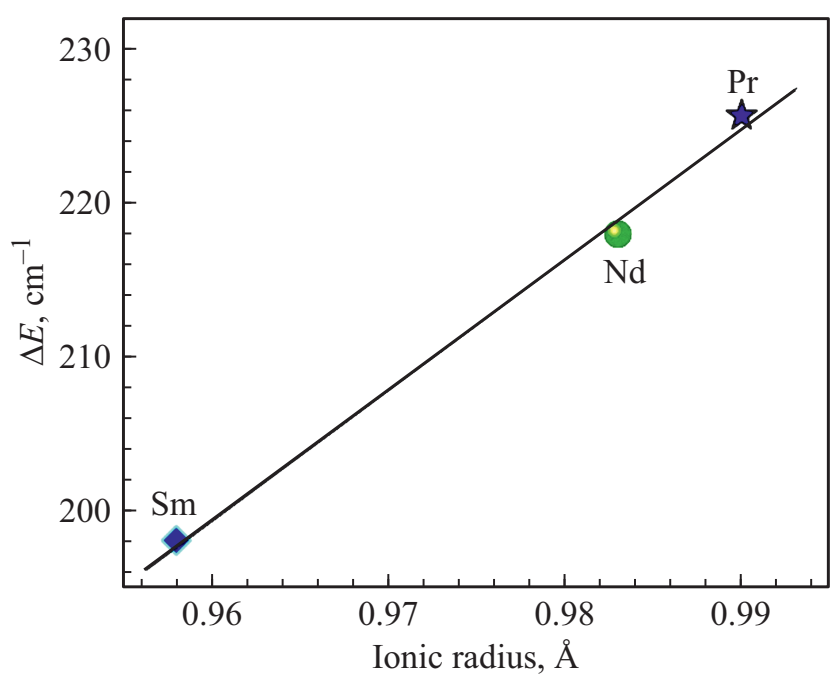

Рис. 3. Зависимость расщепления $\Delta E$ мультиплета ${ }^{4} I_{13 / 2}$ зондового иона $\mathrm{Er}^{3+}$ в кристаллах $R \mathrm{Fe}_{3}\left(\mathrm{BO}_{3}\right)_{4}: \mathrm{Er}^{3+}(R=\mathrm{Pr}$, $\mathrm{Nd}, \mathrm{Sm}$, указаны на рисунке) от ионного радиуса иона $R^{3+}$.

В кристаллическом поле энергетические уровни свободного Р3 иона в общем случае расщепляются на $(2 J+1)$ штарковских уровня $(J-$ полный момент системы). Ион $\mathrm{Er}^{3+}\left(4 f^{11}\right)$ является крамерсовским, и его уровни энергии остаются двукратно вырожденными в отсутствие магнитного поля. При температуре Нееля $T_{\mathrm{N}}=33 \mathrm{~K}$ кристалл $\mathrm{NdFe}_{3}\left(\mathrm{BO}_{3}\right)_{4}$ магнитно упорядочи-

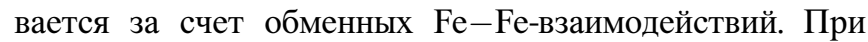
этом в возникающем эффективном магнитном поле происходит снятие крамерсовского вырождения, и каждый штарковский уровень иона эрбия расщепляется на два подуровня. На рис. 2 представлена схема штарковских расщеплений основного ${ }^{4} I_{15 / 2}$ и первого возбужденного ${ }^{4} I_{13 / 2}$ уровней свободного иона $\mathrm{Er}^{3+}$ в кристаллическом поле, а также расщепления крамерсовских дублетов

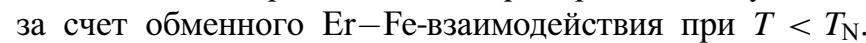
Штарковские уровни основного мультиплета ${ }^{4} I_{15 / 2}$ обозначены арабскими цифрами, а возбужденных - заглавными латинскими буквами.

На схеме обозначен переход 1А со штарковского уровня 1 мультиплета ${ }^{4} I_{15 / 2}$ на уровень А мультиплета ${ }^{4} I_{13 / 2}$. При магнитном упорядочении снимается крамерсовское вырождение дублетов: уровень 1 расщепляется на крамерсовские подуровни $1^{\prime}$ и $1^{\prime \prime}$, а уровень А на $\mathrm{a}^{\prime}$ и $\mathrm{a}^{\prime \prime}$. Тогда спектральная линия, соответствующая переходу 1А, расщепляется в общем случае на четыре компоненты, относящиеся к переходам $1^{\prime} \mathrm{a}^{\prime}, 1^{\prime} \mathrm{a}^{\prime \prime}, 1^{\prime \prime} \mathrm{a}^{\prime}$ и $1^{\prime \prime} \mathrm{a}^{\prime \prime}$. Обменное расщепление основного уровня обозначим $\Delta_{0}$, возбужденного уровня $\mathrm{A}-\Delta_{\mathrm{A}}$.

На рис. 3 приведена зависимость расщепления $\Delta E$ мультиплета ${ }^{4} I_{13 / 2}$ зондового иона $\mathrm{Er}^{3+}$ от ионного радиуса иона $\mathrm{R}^{3+}$ для ферроборатов $R \mathrm{Fe}_{3}\left(\mathrm{BO}_{3}\right)_{4}: \mathrm{Er}^{3+}$ $(R=\mathrm{Sm}, \mathrm{Nd}, \operatorname{Pr}[28])$, имеющих структуру $R 32$. Зависимость $\Delta E(T)$ носит линейный характер. Уменьшение ионного радиуса приводит к уменьшению расстояний $R-\mathrm{O}$ и, следовательно, к увеличению перекрывания волновых функций иона $\mathrm{Er}^{3+}$ и соседних лигандов. Сила кристаллического поля увеличивается, что вызывает увеличение расщепления мультиплетов иона $\mathrm{Er}^{3+}[29,30]$.

Далее обратимся к спектрам зондового иона эрбия в магнитоупорядоченном состоянии ферроборатов самария и неодима. На рис. 4 приведены спектральные линии $1 \mathrm{~A}$ и $1 \mathrm{~B}$ в области перехода ${ }^{4} I_{15 / 2} \rightarrow{ }^{4} I_{13 / 2}$ в ионе $\mathrm{Er}^{3+}$ в $\mathrm{SmFe}_{3}\left(\mathrm{BO}_{3}\right)_{4}: \mathrm{Er}^{3+}$ и $\mathrm{NdFe}_{3}\left(\mathrm{BO}_{3}\right)_{4}: \mathrm{Er}^{3+}$ при различных температурах.

Ниже температуры Нееля $T_{\mathrm{N}}$ упорядоченные магнитные моменты железа создают эффективное магнитное поле на ионах эрбия, что приводит к снятию вырождения штарковских уровней (крамерсовских дублетов) иона $\mathrm{Er}^{3+}$ и расщеплению соответствующих спектральных линий в исследуемых соединениях. Линия $1 \mathrm{~A}$ иона $\mathrm{Er}^{3+}$ в кристаллическом поле ферробората неодима расщепляется на три компоненты, что указывает на одинаковые величины расщепления $\Delta_{0}$ основного состояния и расщепления $\Delta_{\mathrm{A}}$ первого возбужденного уровня А мультиплета ${ }^{4} I_{13 / 2}$.

Для центральной компоненты линии $1 \mathrm{~A}$ иона $\mathrm{Er}^{3+}$ в кристаллическом поле $\mathrm{SmFe}_{3}\left(\mathrm{BO}_{3}\right)_{4}$ наблюдается слабовыраженное плечо, что позволяет разделить линию на четыре компоненты. Обозначения на рис. 4 соответствуют схеме рис. 2. Анализ расщепления спектральных линий 1А при различных температурах позволил определить обменные расщепления основного состояния $\Delta_{0}$ мультиплета ${ }^{4} I_{15 / 2}$ иона $\mathrm{Er}^{3+}$ в $\mathrm{SmFe}_{3}\left(\mathrm{BO}_{3}\right)_{4}$ 

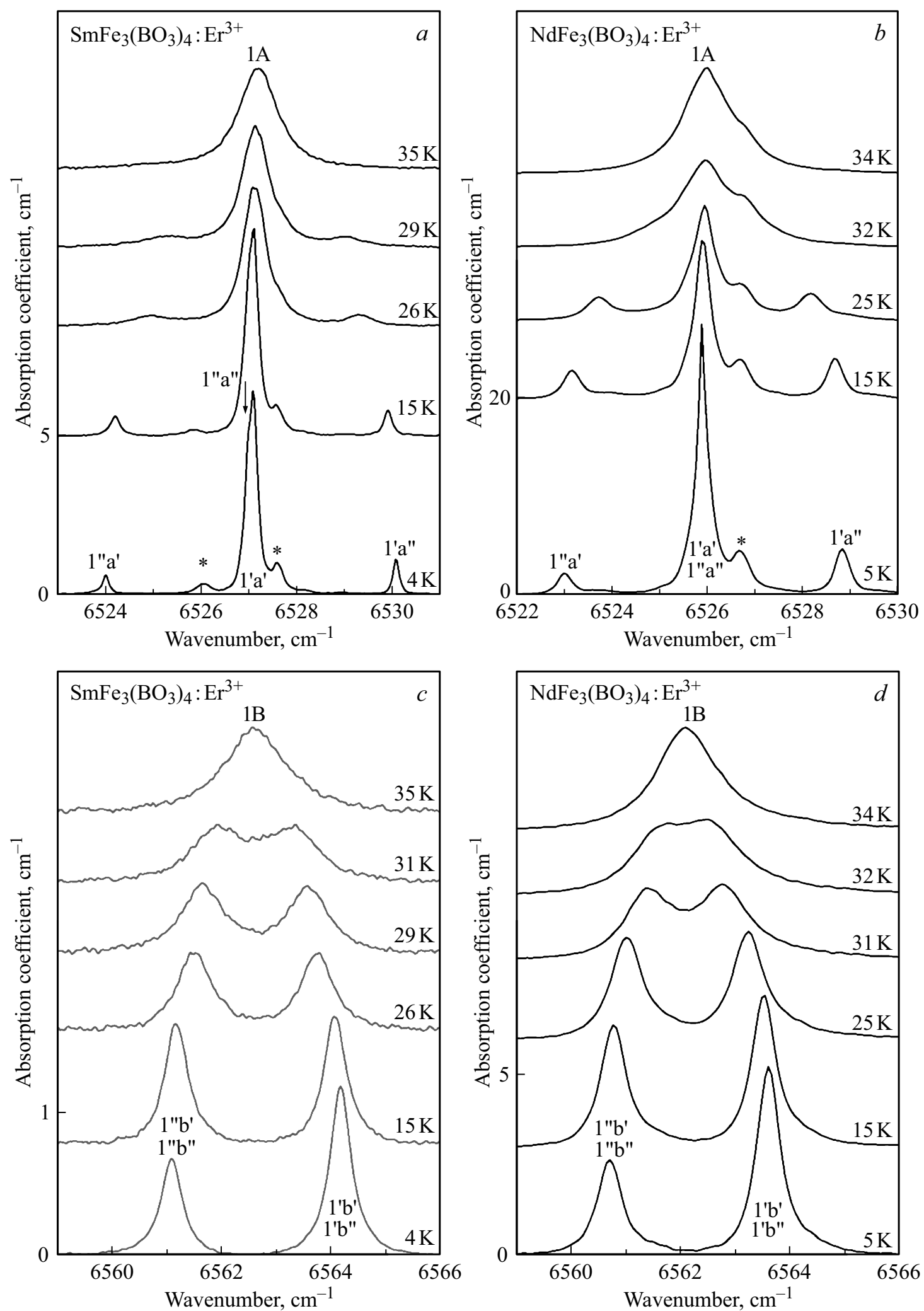

Рис. 4. Линии поглощения $1 \mathrm{~A}(a, b)$ и $1 \mathrm{~B}(c, d)$ в области перехода ${ }^{4} I_{15 / 2} \rightarrow{ }^{4} I_{13 / 2}$ в ионах $\mathrm{Er}^{3+}$ в кристаллах $\mathrm{SmFe}_{3}\left(\mathrm{BO}_{3}\right)_{4}: \mathrm{Er}$ $\left(1\right.$ at.\%) $(a, c)$ и $\mathrm{NdFe}_{3}\left(\mathrm{BO}_{3}\right)_{4}: \operatorname{Er}(1$ at.\%) $(b, d)$. Спектры при разных температурах смещены по вертикали друг относительно друга. Звездочкой $(*)$ обозначены линии примеси. 

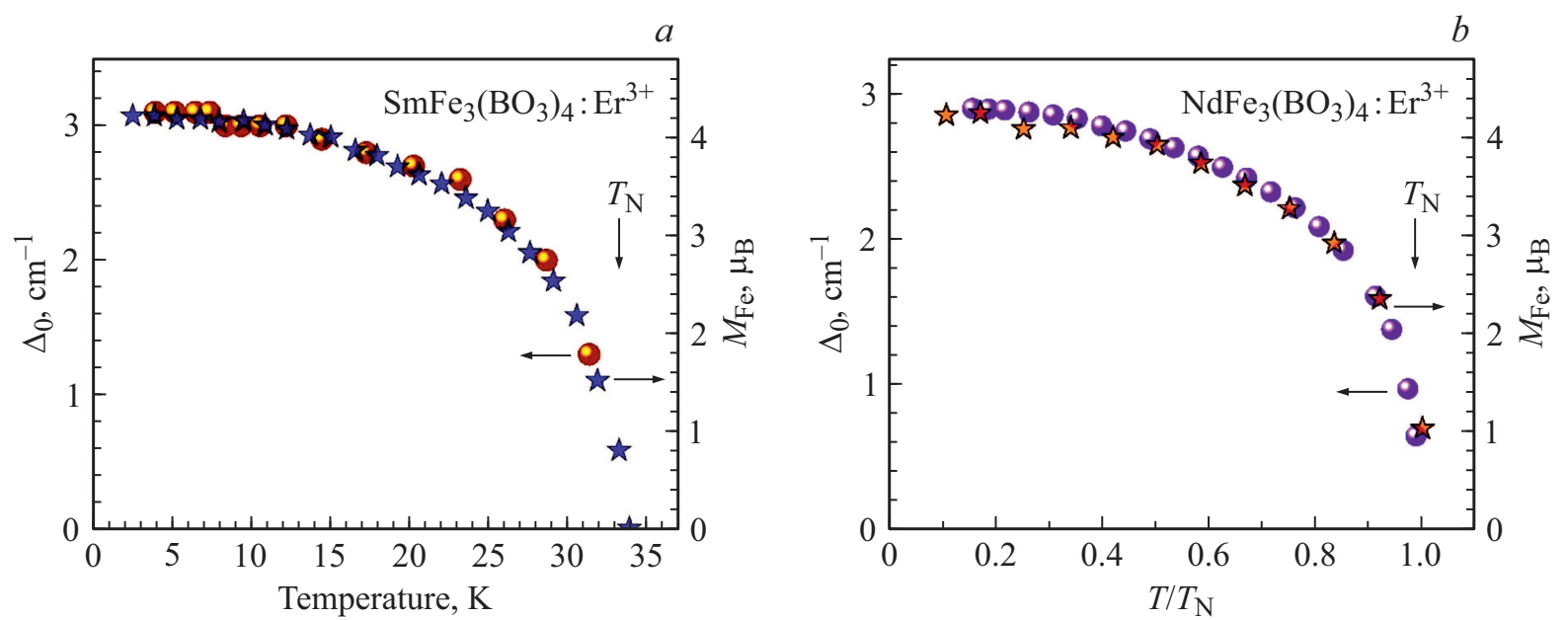

Рис. 5. Температурные зависимости обменного расщепления основного крамерсовского дублета $\Delta_{0}$ иона $\mathrm{Er}^{3+}$ (кружки) в $\mathrm{SmFe}_{3}\left(\mathrm{BO}_{3}\right)_{4}: \mathrm{Er}\left(1\right.$ at.\%) (a) и в $\mathrm{NdFe}_{3}\left(\mathrm{BO}_{3}\right)_{4}: \mathrm{Er}\left(1\right.$ at.\%) (b) и величины магнитных моментов $M_{\mathrm{Fe}}$ ионов $\mathrm{Fe}^{3+}$ (звездочки) по данным измерений рассеяния нейтронов в $\mathrm{SmFe}_{3}\left(\mathrm{BO}_{3}\right)_{4}(a)[19]$ и в $\mathrm{NdFe}_{3}\left(\mathrm{BO}_{3}\right)_{4}(b)$ [26].

Обменные расщепления $\left(\mathrm{cm}^{-1}\right)$ основного состояния $\Delta_{0}$ и уровней А и В первого возбужденного мультиплета ${ }^{4} I_{13 / 2}$ иона $\mathrm{Er}^{3+}-\Delta_{\mathrm{A}}$ и $\Delta_{\mathrm{B}}$ соответственно, в кристаллических полях ферроборатов неодима, самария и празеодима

\begin{tabular}{c|c|c|c}
\hline $\begin{array}{c}\text { Обменное } \\
\text { расщепление } \\
\text { уровня }\end{array}$ & $\mathrm{NdFe}\left(\mathrm{BO}_{3}\right)_{4}$ & $\mathrm{SmFe}\left(\mathrm{BO}_{3}\right)_{4}$ & $\mathrm{PrFe}\left(\mathrm{BO}_{3}\right)_{4}[28]$ \\
\hline$\Delta_{0}$ & 2.9 & 3.08 & 1.49 \\
$\Delta_{\mathrm{A}}$ & 2.9 & 3.01 & 3.9 \\
$\Delta_{\mathrm{B}}$ & $\sim 0.0$ & $\sim 0.0$ & -
\end{tabular}

и $\mathrm{NdFe}_{3}\left(\mathrm{BO}_{3}\right)_{4}$. Полученные результаты в сравнении с данными по $\mathrm{PrFe}_{3}\left(\mathrm{BO}_{3}\right)_{4}$ [28] представлены в таблице.

Линия 1В расщепляется на две компоненты, что свидетельствует о пренебрежимо малом значении расщепления уровня В мультиплета ${ }^{4} I_{13 / 2}$. Это подтверждается тем фактом, что расщепление линии $1 \mathrm{~B}$ при $5 \mathrm{~K}$ равно значению $\Delta_{0}$.

На рис. 5 приведена температурная зависимость $\Delta_{0}(T)$ расщепления основного состояния иона $\mathrm{Er}^{3+}$ для исследуемых соединений. Аппроксимация этой зависимости к нулю дает температуру магнитного упорядочения $T_{\mathrm{N}}=33 \pm 1 \mathrm{~K}$ как для $\mathrm{SmFe}_{3}\left(\mathrm{BO}_{3}\right)_{4}$, так и для $\mathrm{NdFe}_{3}\left(\mathrm{BO}_{3}\right)_{4}$, что неплохо согласуется с литературными данными. Сравнение спектроскопических результатов по расщеплению основного состояния зондового иона $\mathrm{Er}^{3+}$ с данными по величине магнитного момента железа, полученными из экспериментов по рассеянию нейтронов в $\mathrm{SmFe}_{3}\left(\mathrm{BO}_{3}\right)_{4}$ [19] и в $\mathrm{NdFe}_{3}\left(\mathrm{BO}_{3}\right)_{4}$ [26], также представленное на рис. 5 , подтверждает гипотезу эффективного магнитного поля на ионах эрбия, пропорционального магнитному моменту железа $M_{\mathrm{Fe}}$.

Таким образом, обосновывается применимость метода эрбиевого спектроскопического зонда для исследова- ния магнитных фазовых переходов и типов магнитных структур в ферроборатах. Сравним величины обменного расщепления $\Delta_{0}$ основного крамерсовского дублета иона $\mathrm{Er}^{3+}$ при $T=5 \mathrm{~K}$ в исследуемых ферроборатах $\mathrm{NdFe}_{3}\left(\mathrm{BO}_{3}\right)_{4}: \mathrm{Er}$ (1 at.\%) и $\mathrm{SmFe}_{3}\left(\mathrm{BO}_{3}\right)_{4}: \mathrm{Er}$ (1 at.\%), приведенные в таблице, со значением $\Delta_{0}=1.46 \mathrm{~cm}^{-1}$ для легкоосного магнетика $\mathrm{PrFe}_{3}\left(\mathrm{BO}_{3}\right)_{4}: \mathrm{Er}$ (1 at.\%) [28]. Отметим, что все три соединения имеют кристаллическую структуру $R 32$. Величина $\Delta_{0}$ для легкоплоскостных ферроборатов неодима и самария приблизительно в 2 раза больше, чем для легкоосного ферробората празеодима. В проведенных ранее исследованиях типов магнитных структур методом эрбиевого спектроскопического зонда ферроборатов со структурой $P 3_{1} 21$ такое соотношение для $\Delta_{0}$ в соединениях с легкоплоскостной и легкоосной магнитной структурой примерно сохраняется, однако абсолютные значения другие [14], что можно объяснить другой симметрией $\mathrm{P} 3$ центра $\left(D_{3}\right.$ для соединений с пространственной группой $R 32$ и $C_{2}-\mathrm{c}$ группой $\left.P 3_{1} 21\right)$. В отличие от исследованных ранее Р3 ферроборатов со структурой $P 3_{1} 21$ в случае соединений $\mathrm{SmFe}_{3}\left(\mathrm{BO}_{3}\right)_{4}$ и $\mathrm{NdFe}_{3}\left(\mathrm{BO}_{3}\right)_{4}$, имеющих структуру $R 32$, отсутствуют магнитонеэвивалентные позиции для Р3 ионов при температурах ниже $T_{\mathrm{N}}$. В связи с этим спектральные линии, соответствующие поглощению в ионах $\mathrm{Er}^{3+}$, более узкие, чем аналогичные линии в спектрах магнитоупорядоченных ферроборатов со структурой $P 3_{1} 21$.

Поскольку в работе [26] по рассеянию нейтронов в $\mathrm{NdFe}_{3}\left(\mathrm{BO}_{3}\right)_{4}$ говорилось о фазовом переходе в несоразмерную фазу при температуре $T_{\mathrm{IC}}=13.5 \mathrm{~K}$, представляло интерес провести детальное исследование температурных зависимостей характеристик спектральных линий на предмет поиска особенностей, которые могли бы свидетельствовать о дополнительных фазовых переходах. Мы построили температурные зависимости сдвига 

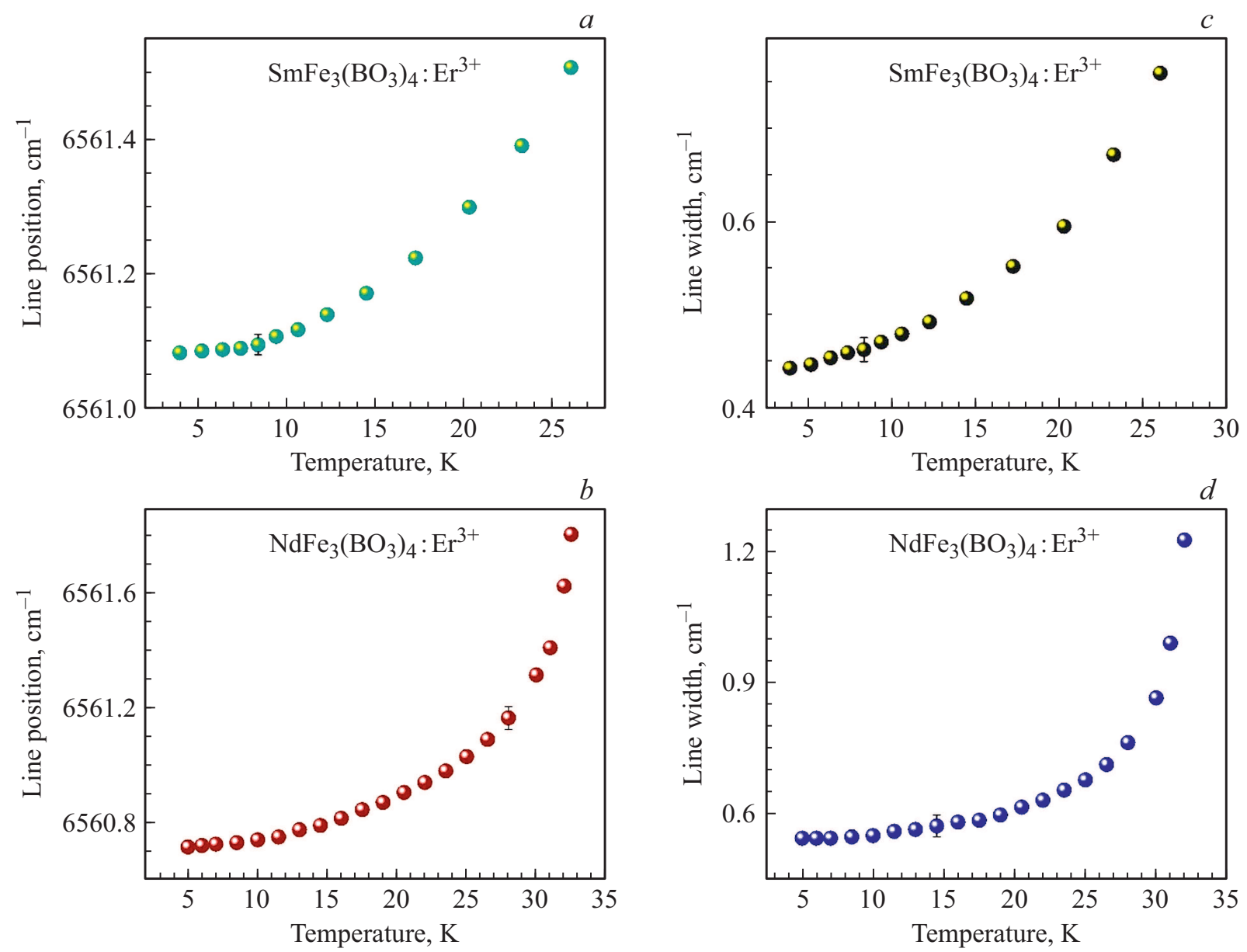

Рис. 6. Температурные зависимости положения $(a, b)$ и полуширины $(c, d)$ низкочастотной компоненты линии $1 \mathrm{~B}$ иона $\mathrm{Er}^{3+}$ в кристаллическом поле $\mathrm{SmFe}_{3}\left(\mathrm{BO}_{3}\right)_{4}: \operatorname{Er}\left(1\right.$ at.\%) $(a, c)$ и $\mathrm{NdFe}_{3}\left(\mathrm{BO}_{3}\right)_{4}: \operatorname{Er}(1$ at.\%) $(b, d)$.

и полуширины низкочастотной компоненты линии $1 \mathrm{~B}$ иона $\mathrm{Er}^{3+}{ }_{\text {в }} \mathrm{SmFe}_{3}\left(\mathrm{BO}_{3}\right)_{4}: \mathrm{Er}\left(1\right.$ at.\%) и $\mathrm{NdFe}_{3}\left(\mathrm{BO}_{3}\right)_{4}: \mathrm{Er}$ (1 at.\%). Они показаны на рис. 6. На представленных зависимостях отсутствуют какие-либо особенности в области температур 13-15K как для $\mathrm{SmFe}_{3}\left(\mathrm{BO}_{3}\right)_{4}$, так и для $\mathrm{NdFe}_{3}\left(\mathrm{BO}_{3}\right)_{4}$. Таким образом, спектры эрбиевого зонда в ферроборатах неодима и самария не позволяют обнаружить какие-либо дополнительные фазовые переходы в этих соединениях.

\section{Заключение}

Исследованы температурные зависимости спектров поглощения в области перехода ${ }^{4} I_{15 / 2} \rightarrow{ }^{4} I_{13 / 2} \quad$ в ионе $\mathrm{Er}^{3+}$ в ферроборатах $\mathrm{NdFe}_{3}\left(\mathrm{BO}_{3}\right)_{4}: \operatorname{Er}(1 \mathrm{at} \%$ и $\mathrm{SmFe}_{3}\left(\mathrm{BO}_{3}\right)_{4}: \mathrm{Er}(1$ at.\%), кристаллическая структура которых описывается пространственной группой $R 32$ вне зависимости от температуры. Оба этих соединения демонстрируют антиферромагнитное упорядочение при $T_{\mathrm{N}}=33 \pm 1 \mathrm{~K}$. Других фазовых переходов не зарегистрировано. Проведено сравнение с данными по легкоосному магнетику $\mathrm{PrFe}\left(\mathrm{BO}_{3}\right)_{4}$, изученному ранее методом эрбиевого спектроскопического зонда. Показано, что метод может быть успешно применен, чтобы различить легкоосный и легкоплоскостной типы магнитного упорядочения в ферроборатах со структурой $R 32$ (ранее такой вывод был сделан относительно ферроборатов со структурой $\left.P 3{ }_{1} 21[12-14]\right)$.

\section{Благодарности}

Работа выполнена на Уникальной Научной Установке (УНУ) ИСАН „Мультифункциональная широкодиапазонная спектроскопия высокого разрешения“ (УНУ МШСВР ИСАН ), http://www.ckp-rf.ru/usu/508571

Авторы благодарны К.Н. Болдыреву за помощь при регистрации части спектров пропускания и С.А. Климину за важные замечания по рукописи. Выражаем благодарность М.Н. Поповой за плодотворное руководство тематикой, мудрое наставничество молодежи, ценные обсуждения и замечания по рукописи.

\section{Финансирование работы}

Работа выполнена при финансовой поддержке Российского научного фонда (грант № 19-12-00413). 


\section{Конфликт интересов}

Авторы заявляют, что у них нет конфликта интересов

\section{Список литературы}

[1] M.N. Popova. J. Alloys and Compounds, 275-277, 142 (1998). DOI: 10.1016/S0925-8388(98)00292-8

[2] M.N. Popova. Encyclopedia of Materials: Science and Technology (Elsevier, 2001), p. 3786-3790.

[3] M.N. Popova, I.V. Paukov, M.N. Popova, Ja. Zoubkova. Sol. State Commun., 79 (7), 569 (1991).

DOI: 10.1016/0038-1098(91)90911-E

[4] M.N. Popova, S.A. Klimin, R. Troć, Z. Bukowski. Solid State Commun., 102 (1), 71 (1997).

DOI: 10.1016/S0038-1098(96)00700-4

[5] N.I. Agladze, G.G. Chepurko, M.N. Popova, E.P. Hlybov. Phys. Lett. A., 133 (4-5), 260 (1988). DOI: $10.1016 / 0375-9601(88) 91028-6$

[6] I.V. Paukov, M.N. Popova, B.V. Mill'. Phys. Lett. A., 169 (1-2), 301 (1992).

DOI: $10.1016 / 0375-9601(92) 90463-\mathrm{V}$

[7] М. Баран, С.А. Климин, Р.3. Левитин, Б.В. Милль, М.Н. Попова, Р. Шимчак. ЖЭТФ, 111 (1), 318 (1997) [M. Baran, R. Szymczak, S.A. Klimin, M.N. Popova, R.Z. Levitin, B.V. Mill'. JETP, 84, 175 (1997). DOI: $10.1134 / 1.558265]$.

[8] Yu.A. Hadjiiskii, R.Z. Levitin, B.V. Mill', I.V. Paukov, M.N. Popova, V.V. Snegirev. Solid State Commun., 859), 743 (1993). DOI: 10.1016/0038-1098(93)90663-8

[9] Yu.A. Hadjiiskii, I.V. Paukov, M.N. Popova, B.V. Mill'. Phys. Lett. A., 189 (1-2), 109 (1994).

DOI: 10.1016/0375-9601(94)90827-3

[10] M.N. Popova, Yu.A. Hadjiiskii, I.V. Paukov, B.V. Mill'. Phys. Lett. A., 203 (5-6), 412 (1995).

DOI: $10.1016 / 0375-9601(95) 00391-F$

[11] S.A. Klimin, A.S. Galkin, M.N. Popova. Phys. Lett. A., 376 (23), 1861 (2012). DOI: 10.1016/j.physleta.2012.03.013

[12] M.N. Popova, E.P. Chukalina, T.N. Stanislavchuk, L.N. Bezmaternykh. JMMM, 300 (1), e440-e443 (2006). DOI: $10.1016 /$ j.jmmm.2005.10.187

[13] T.N. Stanislavchuk, E.P. Chukalina, M.N. Popova, L.N. Bezmaternykh, I.A. Gudim. Phys. Lett. A., 368 (5), 408-411 (2007). DOI: 10.1016/j.physleta.2007.04.044

[14] M.N. Popova. JMMM, 321 (7), 716 (2009). DOI: $10.1016 /$ j.jmmm.2008.11.033

[15] E.P. Chukalina, M.N. Popova, L.N. Bezmaternykh, I.A. Gudim. Phys. Lett. A., 374 (15-16), 1790 (2010). DOI: $10.1016 /$ j.physleta.2010.02.018

[16] А.М. Кадомцева, Ю.Ф. Попов, Г.П. Воробьев, А.П. Пятаков, С.С. Кротов, К.И. Камилов, В.Ю. Иванов, А.А. Мухин, А.К. Звездин, А.М. Кузьменко, Л.Н. Безматерных, И.А. Гудим, В.Л. Темеров. Физ. Низк. Темп., 36 (6), 640 (2010). [A.M. Kadomtseva, Yu.F. Popov, G.P. Vorob'ev, A.P. Pyatakov, S.S. Krotov, K.I. Kamilov, V.Yu. Ivanov, A.A. Mukhin, A.K. Zvezdin, A.M. Kuz'menko, L.N. Bezmaternykh, I.A. Gudim, V.L. Temerov. Low Temp. Phys., 36 (6), 511 (2010). DOI: 10.1063/1.3457390].

[17] А.А. Мухин, Г.П. Воробьев, В.Ю. Иванов, А.М. Кадомцева, А.С. Нарижная, А.М. Кузьменко, Ю.Ф. Попов, Л.Н. Безматерных, И.А. Гудим. Письма в ЖЭТФ, 93 (5), 305 (2011) [A.A. Mukhin, G.P. Vorob'ev, V.Yu. Ivanov,
A.M. Kadomtseva., A.S. Narizhnaya, A.M. Kuz'menko, Yu.F. Popov, L.N. Bezmaternykh, I.A. Gudim. JETP Letters., 93 (5), 275 (2011). DOI: 10.1134/S0021364011050079].

[18] Ю.Ф. Попов, А.П. Пятаков, А.М. Кадомцева, Г.П. Воробьев, А.К. Звездин, А.А. Мухин, В.Ю. Иванов, И.А. Гудим. ЖЭТФ, $138(2), 226$ (2010). [Yu.F. Popov, A.P. Pyatakov, A.M. Kadomtseva, G.P. Vorob'ev, A.K. Zvezdin, A.A. Mukhin, V.Yu. Ivanov, I.A. Gudim. JETP, 111 (2), 199 (2010). DOI: 10.1134/S1063776110080066].

[19] C. Ritter, A. Pankrats, I. Gudim, A. Vorotynov. J. Phys.: Condens. Matter., 24 (38), 386002 (2012). DOI: $10.1088 / 0953-8984 / 24 / 38 / 386002$

[20] М.Н. Попова, Е.П. Чукалина, Б.З. Малкин, Д.А. Ерофеев, Л.Н. Безматерных, И.А. Гудим. ЖЭТФ, $145(1)$, 128 (2014). DOI: 10.7868/S0044451014010131 [M.N. Popova, E.P. Chukalina, B.Z. Malkin, D.A. Erofeev, L.N. Bezmaternykh, I.A. Gudim. JETP, 118 (1), 111 (2014). DOI: $10.1134 / \mathrm{S} 1063776114010142]$.

[21] J.A. Campa, C. Cascales, E. Gutierrez-Puebla, M.A. Monge, I. Rasines, C. Ruiz-Valero. Chem. Mater., 9(1), 237 (1997). DOI: $10.1021 / \mathrm{cm} 960313 \mathrm{~m}$

[22] E.P. Chukalina, D.Yu. Kuritsin, M.N. Popova, L.N. Bezmaternykh, S.A. Kharlamova, V.L. Temerov. Phys. Lett. A., 322 (3-4), 239 (2004). DOI: $10.1016 /$ j.physleta.2003.12.062

[23] N. Tristan, R. Klinger, C. Hess, B. Buchner, E. Popova, I.A. Gudim, L.N. Bezmaternykh. JMMM, $316(2)$, e621 (2007). DOI: 10.1016/j.jmmm.2007.03.046

[24] M.N. Popova, E.P. Chukalina, T.N. Stanislavchuk, B.Z. Malkin, A.R. Zakirov, E. Antic-Fidancev, E.A. Popova, L.N. Bezmaternykh, V.L. Temerov. Phys. Rev. B., 75 (22), 224435 (2007). DOI: 10.1103/PhysRevB.75.224435

[25] P. Fisher, V. Pomjakushin, D. Sheptyakov, L. Keller, M. Janoschek, B. Roessli, J. Schefer, G. Petrakovskii, L. Bezmaternikh, V. Temerov, D. Velikanov. J. Phys.: Condens. Matter., 18 (34), 7975 (2006).

DOI: $10.1088 / 0953-8984 / 18 / 34 / 010$

[26] M. Janoschek, P. Fischer, J. Schefer, B. Roessli, V. Pomjakushin, M. Meven, V. Petricek, G. Petrakovskii, L. Bezmaternikh. Phys. Rev. B., 81 (9), 094429 (2010). DOI: 10.1103/PhysRevB.81.094429

[27] I.A. Gudim, E.V. Eremin, V.L. Temerov. J. Crystal Growth., 312 (16-17), 2427 (2010).

DOI: $10.1016 /$ j.jcrysgro.2010.05.013

[28] M.N. Popova, T.N. Stanislavchuk, B.Z. Malkin, L.N. Bezmaternykh. Phys. Rev. B., 80 (19), 195101 (2009). DOI: 10.1103/PhysRevB.80.195101

[29] P. Caro, O. Beaury, E. Antic. J. de Physique, 37 (6), 671 (1976).

[30] E. Antic-Fidancev, M. Lemaitre-Blaise, P. Caro. New J. Chem., 11 (6), 467 (1987). 